\title{
Phase Noise Suppression in Interferometric Radar Data using Goldstein Noise Filtration
}

\author{
Andrey V. Sosnovsky ${ }^{1,}$ a), Nina S. Vinogradova ${ }^{1, \text { b) }}$ \\ ${ }^{1}$ Yeltsin Ural Federal University, Yekaterinburg, Russia \\ a) Corresponding author: sav83@e1.ru \\ b) n.s.vinogradova@urfu.ru
}

\begin{abstract}
The article is devoted the coherence maps utilization for the phase noise filtration using the Goldstein filter for interferometric synthesized aperture radar (InSAR) images. For the ALOS PALSAR data (FBS imaging mode), four coherence estimation techniques (classical, difference slope compensation, Fourier slope compensation, and «peak-Fourier») were researched as the Goldstein filter parameter. The filtered interferograms were compared with the reference ground control points reprojected into the radar coordinate system. It is shown that the coherence estimation method affects the quality of the phase noise suppression.
\end{abstract}

Keywords: Interferometric radar images, coherence maps, spectral adaptive image filters.

\section{INTRODUCTION}

Nowadays, the use of images obtained by the synthesized aperture radars (SAR) is of wide interest, since such data do not depend on the time of day and weather conditions [1-5]. This is especially urgent for the most of Russian territory located in the northern regions and the most part of the year hidden by clouds. Essence of any problem solved by using the SAR data is the correct construction of a difference-phase image, or the interferogram. At the first stage of the interferogram generation, the images of the pair are spatially aligned, while the second (slave) image is recalculated into the geometry of the first (master) one. Due to influence of the atmospheric effects and terrain features, the interferogram is distorted under the influence of the phase noise; so, it is mandatory to filter it. Then, the filtered interferogram is transformed into a matrix of the absolute phase values (unwrapped phase). The final product of the interferogram processing is calculation of the digital elevation model (DEM) or the displacement map. One of the indicators of the obtained interferogram quality is the matrix, which is a field of correlation coefficients between the master and slave SAR images; it is called the coherence map. The higher the value of the brightness elements at the coherence map, the more accurate the output interferogram will be. The spectral adaptive image filter (Goldstein filter, [6]) utilizes the coherence map for the spectral window width manipulation. The present paper focuses on analyzing the coherence map calculation methods for improving the accuracy of the output interferogram.

\section{COHERENCE MAPS AND GOLDSTEIN FILTRATION}

The coherence map is an image of the SAR pair correlation coefficients field. It gives information about deviation degree of the absolute phase from its true value. Such deviation may be caused by the phase noise, a surface variability, and the instability of the radio signal reflected from various parts of the Earth surface [1]. The coherence map may be useful for a wide range of problems solved by systems of the radar remote sensing data such as the urban planning, emergencies monitoring, and issues of environmental protection. It allows one to view the characteristics of the satellite system, atmospheric and weather conditions, the properties of the radar signal and the

International Conference of Numerical Analysis and Applied Mathematics ICNAAM 2019

AIP Conf. Proc. 2293, 140023-1-140023-4; https://doi.org/10.1063/5.0027278 Published by AIP Publishing. 978-0-7354-4025-8/\$30.00 
Earth surface. But, first of all, to assess the quality of products created on the basis of SAR data, such as the DEM and terrain displacement maps [1,3,5]. The coherence map calculation is performed after the stage of the SAR interferogram generation or interferogram attending. According to the traditional technique, each element is calculated as the correlation coefficient between the values of the first and second SAR images in the pair

$$
\gamma=\frac{\left|\sum_{m, n} \dot{Z}_{1}(m, n) \bar{Z}_{2}(m, n)\right|}{\sqrt{\sum_{m, n}\left|\dot{Z}_{1}(m, n)\right|^{2} \cdot \sum_{m, n}\left|\dot{Z}_{2}(m, n)\right|^{2}}},
$$

where $Z_{1}, Z_{2}$ are the radar images of the reference and auxiliary signals respectively. The classical method of the coherence map development is based on multiplying the first (reference) image of the interferometric pair by the second (auxiliary) that is complex-conjugate to itself.

However, this approach causes the problem of degradation of the estimate, because a random variable instead of a random process is estimated here. So, any phase slopes cause a decrease of the estimate to the values about zero [7]. To eliminate this problem, some modifications of estimation were offered [7]. The first approach uses the phase slope estimation instead of phase itself:

$$
\gamma=\frac{\left|\sum_{m, n} \dot{w}_{1}(m, n) \bar{w}_{2}(m, n)\right|}{\sqrt{\sum_{m, n}\left|\dot{w}_{1}(m, n)\right|^{2} \cdot \sum_{m, n}\left|\dot{w}_{2}(m, n)\right|^{2}}}, \quad \dot{w}_{1,2}(m, n)=\dot{Z}_{1,2}(m, n) \cdot \bar{Z}_{1,2}(m-1, n) .
$$

The second uses the 2D fast Fourier transform (2FFT) to determine the complex interferogram modulation frequencies, which can be found as

$$
\left[\omega_{x}, \omega_{y}\right]=\arg \max \left\{F\left[\dot{Z}_{1} \bar{Z}_{2}\right]\right\},
$$

where $\omega_{x}$ and $\omega_{y}$ are the spatial frequencies in both spatial dimensions, which are estimated in the interferogram sample of $M \times N$ size; $F\{\}$ is the 2D Fourier transform. Then, the spatial frequency demodulation is performed

$$
\dot{Z}_{2 c}=\dot{Z}_{2} \exp \left[-j\left(\frac{\omega_{x}}{M} m+\frac{\omega_{y}}{N} n\right)\right],
$$

and then coherence is calculated by formula (1) with $\dot{Z}_{2 c}$ instead of $\dot{Z}_{2}$. To improve the computational efficiency of the Fourier slope estimate, the normalized spectrum peak value proportional and biased to $P /(M \times N)$ value can be used as a coherence estimate instead of the correlation coefficient, where $P$ is the peak height in the spectral domain. The way for the DEM accuracy improvement with the coherence maps implies their application as an adaptation parameter for the phase noise filters to suppress the phase noise.

The spectral adaptive filter (Goldstein filter with the Baran modification) is the most widespread among the phase noise filters for InSAR data. It uses the two-dimensional FFT spectra $F(k, l)$ calculated in the local windows, which then are weighed in the following way:

$$
\dot{I}^{F}(m, n)=\mathbb{F}^{-1}\left\{|\dot{F}(k, l)|^{\alpha(1-\overline{\gamma(k, l)})} \cdot \dot{F}(k, l)\right\},
$$

where $\overline{\gamma(k, l)}$ is the coherence estimate averaged in the filter window; $\alpha$ is the scaling coefficient, which is often used to improve the filter performance.

The filter operates with overlapping windows, and the samples of the filtered interferogram $\dot{I}^{F}(m, n)$, which lay in the overlapped areas, are summed with the triangular weighting function. Also, an additional mean filtration may be used to smooth the weighting function $|\dot{F}(k, l)|$. Different coherence estimators $\gamma$ may give different suppression results; so, it should be reasonable to examine different estimators mentioned above. 


\section{EXPERIMENTAL RESULTS}

Experimental imagery data were received by the ALOS PALSAR radar (wavelength $1.27 \mathrm{GHz}$, radiometric resolution 5 bits) and were represented by the interferometric pair of SAR images for the polygon contained different surface types (fields, forests, urban, and mining areas). The phase field has an ambiguity height of $62 \mathrm{~m}$; the spatial sampling interval was $7 \times 3 \mathrm{~m}$, and the scene size was $20000 \times 10000$ samples. The technique for experimental accuracy estimation of the processed InSAR data implies calculation of the standard deviation for the imagery data and reference one in the radar coordinate system. The reference data were represented by the set of 2042 reference height points with a vertical accuracy of $0.5 \mathrm{~m}$, and contained average hills and river valleys. The coordinates of the reference height points were transformed into the WGS- 84 coordinate system and then reprojected into the radar coordinate system. For investigation of DEM accuracy improvement after the phase noise suppression, the following spectral adaptive filter parameters and adjustments were examined:

— the filtering block size was $25 \times 25,40 \times 40$ and $55 \times 55$;

- the coherence estimation type: classical, Fourier slope compensation, and Fourier spectral peak one;

- the coherence estimation window size varied from $3 \times 3$ to $31 \times 31$.

— the filter scaling coefficient $\alpha$ varied from 2.5 to 4.5 in order to improve the filter efficiency.

The results are presented in Fig. 1. At first, the filter without the coherence map application $(\bar{\gamma}=0)$ was examined for different filter block sizes, and the best $\alpha$-values for each block size were determined (Fig. 1a): $\alpha_{25}=4.0, \alpha_{40}=3.0$, and $\alpha_{55}=3.5$. Then, the $\alpha$ was adjusted for each filtering procedure in the following way:

$$
\alpha=\frac{\alpha_{i}}{1-\bar{\gamma}_{M i j}},
$$

where $\alpha_{i}$ is $\alpha_{25}, \alpha_{40}$, or $\alpha_{55}, \bar{\gamma}_{M i j}$ is the mean coherence of the coherence map, calculated with $j$-th window halfsize $(j=\{1,3,7,11,15\})$ and applied for the $i$-th filter block size $(i=\{25,40,55\})$. It is seen that the use of coherence maps in the suppression of the phase noise in many cases leads to a filtration accuracy improvement and is expressed in a decrease of the relative phase standard deviation (STD). Classical, slope-Fourier and peak-Fourier estimates behave themselves broadly similar for the coherence window half-sizes more than $3 \times 3$ (the peak-Fourier estimate fails for small window sizes), and apart from the phase slope coherence estimate. The last gives the best results in phase filtering, decreasing the STD by a value about of $0.01 \mathrm{rad}$ (or $0.1 \mathrm{~m}$ of for the vertical height STD). But the result is not significant, so the further improvement in coherence estimation techniques for better phase noise filtration is needed.

\section{CONCLUSION}

The methods of the coherence map calculation for implementation in Goldstein interferogram phase noise filter were investigated in order to improve the accuracy of the output interferogram. It is shown that the application of coherence maps can improve the accuracy of the phase noise suppression results only by 0.01 rad, which corresponds to the height accuracy improvement of $0.1 \mathrm{~m}$. The best suppression is achieved by using the phase slope coherence estimation. The result is not significant, so the further improvement in coherence estimation techniques for better phase noise filtration is needed. 


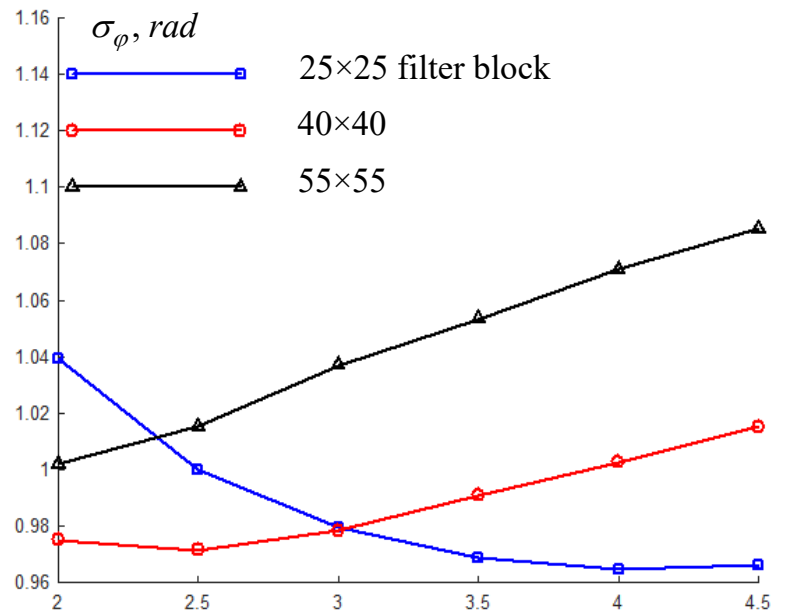

(a)

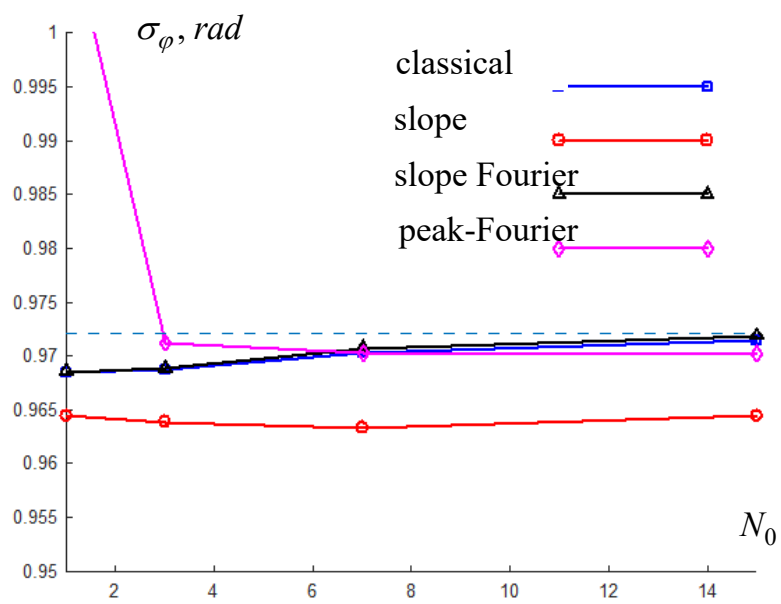

(c)

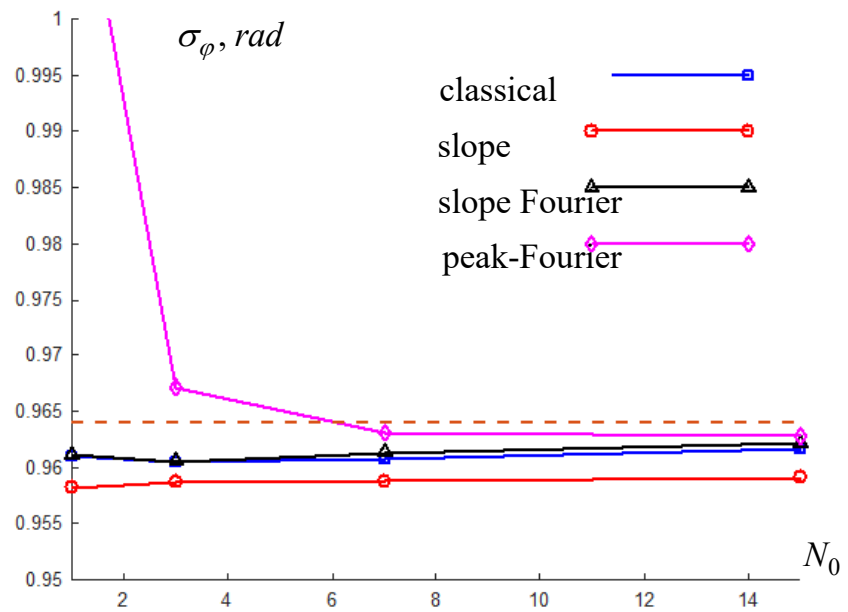

(b) $\alpha$

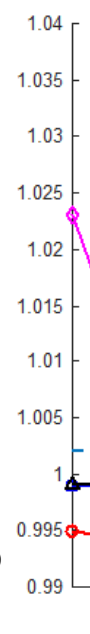

$\sigma_{\varphi}, \mathrm{rad}$

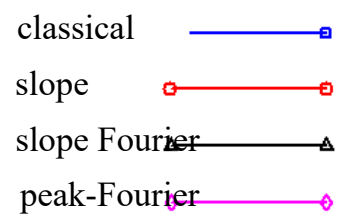

FIGURE 1. Dependence of the relative phase STD after the adaptive spectral filter (radians) from the Goldstein filter without coherence estimation (a) and for coherence estimation window half-size $N_{0}\left(N=M=2 N_{0}+1\right)$. Cases: b) the size of the filtration block is $25 \times 25$, c) block size is $40 \times 40$ d) block size is $55 \times 55$

\section{ACKNOWLEDGMENTS}

This work was supported by the Center of Excellence "Geoinformation technologies and geophysical data complex interpretation" of the Ural Federal University Program (Act 211 Government of the Russian Federation, contract № 02.A03.21.0006).

\section{REFERENCES}

1. A. Monti Guarnieri, P. Guccione, P. Pasquali, and Y.L. Desnos, IEE Proc. Radar Sonar Navig., 150(3), 193-200 (2003).

2. I.R. Joughin, F.K. Li, S.N. Madsen, and E. Rodrigues, Proceedings of the IEEE, 88(3), 333-382 (2000).

3. R. Touzi, A. Lopes, and P. W. Vachon, IEEE Transactions on Geoscience and Remote Sensing, 37(1), 135-149 (1999).

4. L.G. Dorosinsky. "The research of the distributed objects' radar image recognition algorithms" in 23rd International Crimean Conference Microwave and Telecommunication Technology "CRIMICO-2013», Conference Proceedings vol. 2 (Sevastopol, Crimea; Ukraine, 2013), pp. 1216-1217.

5. M. Cattabeni, A. Monti-Guarnieri, and F. Rocca. "Estimation and Improvement of Coherence in SAR Interferograms" in Geoscience and Remote Sensing Symposium, 1994. IGARSS '94. Surface and Atmospheric Remote Sensing: Technologies, Data Analysis and Interpretation. Vol. 4 (IEEE, 1994) pp. 720-722.

6. I.A. Baran, M.P. Stewart, B.M. Kampes, Z. Persky, and P. Lilli, IEEE Trans. Geosci. Rem. Sens. 41(9), $2114-2118$ (2003).

7. A.V. Sosnovsky, V.G. Kobernichenko, N.S. Vinogradova, and O. Tsogtbaatar, CEUR-WS 1814, 98-105 (2017). 